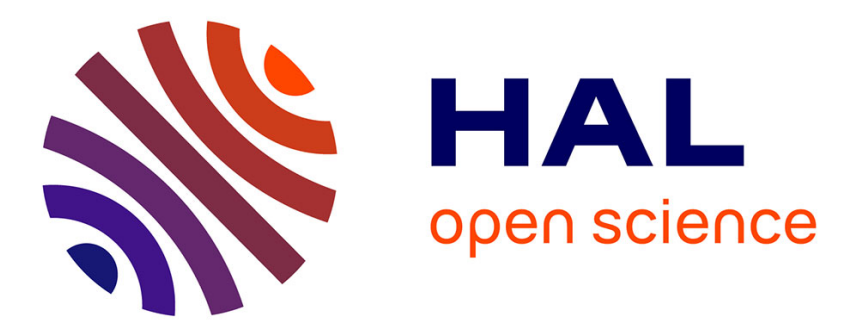

\title{
Structure-activity analysis of aging and reactivation of human butyrylcholinesterase inhibited by analogues of tabun
}

\author{
Eugénie Carletti, Nadine Aurbek, Emilie Gillon, Mélanie Loiodice, Yvain \\ Nicolet, Juan-Carlos Fontecilla-Camps, Patrick Masson, Horst Thiermann, \\ Florian Nachon, Franz Worek
}

\section{To cite this version:}

Eugénie Carletti, Nadine Aurbek, Emilie Gillon, Mélanie Loiodice, Yvain Nicolet, et al.. Structureactivity analysis of aging and reactivation of human butyrylcholinesterase inhibited by analogues of tabun. Biochemical Journal, 2009, 421 (1), pp.97-106. 10.1042/BJ20090091 hal-00479152

\section{HAL Id: hal-00479152 https://hal.science/hal-00479152}

Submitted on 30 Apr 2010

HAL is a multi-disciplinary open access archive for the deposit and dissemination of scientific research documents, whether they are published or not. The documents may come from teaching and research institutions in France or abroad, or from public or private research centers.
L'archive ouverte pluridisciplinaire HAL, est destinée au dépôt et à la diffusion de documents scientifiques de niveau recherche, publiés ou non, émanant des établissements d'enseignement et de recherche français ou étrangers, des laboratoires publics ou privés. 
Structure-activity analysis of aging and reactivation of human butyrylcholinesterase inhibited by analogues of tabun.

\section{Eugénie CARLETTI*, Nadine AURBEK ${ }^{\dagger}$, Emilie GILLON*, Mélanie LOIODICE*, Yvain NICOLET ${ }^{\ddagger}$, Juan-Carlos FONTECILLA-CAMPS ${ }^{\ddagger}$, Patrick MASSON*, Horst THIERMANN ${ }^{\dagger}$, Florian NACHON* and Franz WOREK ${ }^{\dagger}$}

\footnotetext{
* Unité d'Enzymologie, Département de Toxicologie, Centre de Recherches du Service de Santé des Armées (CRSSA), 24 av des Maquis du Grésivaudan, 38700 La Tronche, France.

$\dagger$ Bundeswehr Institute of Pharmacology and Toxicology, Neuherbergstrasse 11, 80937 Munich, Germany.

* Laboratoire de Cristallogenèse et Cristallographie des Protéines. Institut de Biologie Structurale (CEA-CNRS-UJF). 41 rue Jules Horowitz, 38027 Grenoble, France.
}

Running title. Structural analysis of phosphoramidyl-hBChE conjugates.

Corresponding author:

Dr. Florian Nachon,

Unité d'enzymologie, Département de Toxicologie,

Centre de Recherches du Service de Santé des Armées (CRSSA),

24 av des Maquis du Grésivaudan, 38700 La Tronche, France.

Tel: +33476639765 / Fax: +33476636962

E-mail: fnachon@crssa.net 


\begin{abstract}
Human butyrylcholinesterase (hBChE) naturally scavenges organophosphorus nerve agents (OPs). This bioscavenger is currently in Clinical Phase I for pretreatment of OP intoxication. Phosphylated cholinesterases (ChEs) can undergo a spontaneous time-dependent process called «aging" during which the conjugate is dealkylated, leading to non-reactivable enzyme. hBChEs inhibited by phosphoramidates like tabun display a peculiar resistance to oximes mediated reactivation. We investigated the basis of oxime resistance of phosphoramidyl-BChE conjugates, by determining the kinetics of inhibition, reactivation (obidoxime, TMB-4, HLö 7, HI-6), aging, and the crystal structures of hBChE inhibited by different N-monoalkyl and N,N-dialkyl tabun analogues. The refined structures of aged $\mathrm{hBChE}$ conjugates show that aging proceeds through O-dealkylation of the $\mathrm{P}(\mathrm{R})$ enantiomer of N,N-diethyl and N-propyl analogues, with subsequent formation of a salt bridge preventing reactivation, similarly to previous observation made on tabun-ChEs conjugates. Interestingly, the N-methyl analogue projects its amino group towards the choline-binding pocket, so that aging proceeds through deamination. This orientation results from a preference of hBChE's acyl-binding pocket for larger than 2-atoms linear substituents. The correlation between the inhibitory potency and the N-monoalkyl chain length is related to increasingly optimized interactions with the acyl-binding pocket as shown by the X-ray structures. These kinetics and $\mathrm{X}$-ray data lead to a structure-activity relationship that hightlights steric and electronic effects of the amino substituent of phosphoramidate. This study provides structural basis to design new oximes capable of reactivating phosphoramidyl-hBChE conjugates after intoxication, notably when $\mathrm{hBChE}$ is used as pretreatment or to design BChE-based catalytic bioscavengers.
\end{abstract}

Key words: Butyrylcholinesterase, tabun analogues, X-ray crystallography, kinetics, aging, oxime reactivation. 
Abbreviations used: AChE, Acetylcholinesterase; BChE, Butyrylcholinesterase; BSA, Bovine serum albumine; BTC, butyrylthiocholine; ChE, Cholinesterase; $\mathrm{CHO}$, Chinese hamster ovary; DTNB, 5, 5'-dithiobis-(2-nitrobenzoic-acid); HI-6, 1-[[[4-(aminocarbonyl) pyridinio] methoxy] methyl]-2-[(hydroxyimino)methyl]pyridinium dichloride monohydrate; HLö 7, 1[[[4-(aminocarbonyl) pyridinio]methoxy]methyl]-2,4-bis-[(hydroxyimino)methyl] pyridinium dimethanesulfonate); MES, 2-(N-morpholino)ethanesulfonic acid; obidoxime, 1,1'-(oxybismethylene) bis[4-(hydroxyimino) methyl] pyridinium dichloride; OP, Organophosphate; TFA, Trifluoroacetic Acid; TMB-4, 1,3-trimethylene-bis(4-hydroxyiminomethylpyridinium) dibromide. 


\section{Introduction}

Human plasma butyrylcholinesterase (BChE; EC 3.1.1.8) is a toxicologically relevant enzyme because it hydrolyzes or scavenges a wide range of toxic esters, including heroin, cocaine, carbamate pesticides, organophosphorus pesticides and nerve agents [1, 2]. Organophosphates (OPs) exert their acute toxicity through inhibition of acetylcholinesterase (AChE, E.C. 3.1.1.7) by phosphylation of the catalytic serine [2]. Subsequent accumulation of acetylcholine at neuronal synapses and neuromuscular junctions, results in paralysis, seizures and other symptoms of cholinergic syndrome [3, 4]. Human $\mathrm{BChE}$ is a potential drug candidate that may be employed in several therapeutic fields. This is due to the unique structure of the enzyme that allows accommodation of a large variety of substrates and inhibitors, some of which are excluded from the catalytic gorge of acetylcholinesterase [5]. BChE-based biopharmaceuticals are under research and development as stoichiometric and catalytic bioscavengers for neutralization or detoxification of poisonous esters. Recombinant and plasma-purified $\mathrm{hBChE}$, are in Clinical Phase I as the first stoichiometric bioscavengers for the pretreatment of OPs intoxication. In case of intoxication, reactivation of hBChE used in pretreatment will improve protection against high dose or repetitive OP exposure. For this reason it is essential to design efficient reactivators for $\mathrm{hBChE}$.

Phosphylated ChEs can be reactivated by specific nucleophiles such as oximes $[6,7]$. The most effective oximes used for emergency treatment of nerve agent poisoning are the monopyridinium oximes (2-PAM) and bispyridinium oximes (TMB-4, MMB-4, obidoxime, HI-6 and HLö 7) [6]. Kinetic analysis of the interactions between different AChE-OP conjugates and different oximes shows that the most effective oximes are HLö 7 for phosphonylated AChE and obidoxime for phosphorylated AChE [6]. Unfortunately, a universal antidote, efficient against all nerve agents and capable of crossing the blood brain barrier, is not yet available.

Reactivation of inhibited ChEs is complicated by a side reaction. Indeed, phosphylated ChEs undergo a spontaneous, time-dependent process called «aging » during which the OPChE conjugate are O-dealkylated, leading to non-reactivatable enzyme [8-10].

$\mathrm{BChE}$ inhibited by tabun and subsequent aging reaction of adduct are of interest, because tabun-ChE conjugates are particulary resistant to most oxime reactivators. Recent studies detail the mode of interaction of tabun with ChEs and show that tabun-ChE conjugates age through O-dealkylation [11]. An extension of this study to various phosphoramidate anologues of tabun provide the basis of a structure-activity relationship analysis of 
phosphoramidate adducts. The analogues of tabun differs in their amino substituents (Fig. 1). The reactivation of $\mathrm{hBChE}$ inhibited by tabun and its analogues was studied with obidoxime, TMB-4, HI-6 and HLö-7 (Fig. 2). This study provide a better understanding of the resistance of phosphoramidate-hBChE conjugates to oximes and give some insights to design new oximes capable of reactivating tabun-ChEs conjugates, and ChEs inhibited by related agents.

\section{Material and Methods}

\section{Materials}

5,5'-dithio-bis-2-nitrobenzoic acid (DTNB) and TMB-4 dibromide (TMB-4) were obtained from Sigma Chemical Co (St Louis, USA). Obidoxime dichloride (obidoxime) was purchased from Duphar. HI-6 dichloride (HI-6) was kindly provided by Dr. Clement (DRDC, Suffield, Canada) and HLö-7 dimethane-sulfonate (HLö-7) was a custom synthesis by Dr. Braxmeier (Chemisches Labor, Döpshofen, Germany). Tabun and its analogues (TA 1-6) (>98\% by GCMS, ${ }^{1} \mathrm{H}-\mathrm{NMR}$ and $\left.{ }^{31} \mathrm{P}-\mathrm{NMR}\right)$ were made available by the German Ministry of Defence, all other chemicals were from Merck Eurolab GmbH. S-butyrylthiocholine iodide (BTCh) was obtained from Sigma.

Stock solutions of tabun and compounds $1-6(1 \% \mathrm{v} / \mathrm{v}$; Fig. 1) were prepared in 2-propanol, stored at $4^{\circ} \mathrm{C}$ and were appropriately diluted in distilled water just before the experiment. Oximes $(200 \mathrm{mM})$ were prepared in distilled water, stored at $-60^{\circ} \mathrm{C}$ and diluted as required in distilled water at the day of the experiment. Human plasma was obtained from heparinized whole blood by centrifugation ( $3000 \mathrm{rpm}, 10 \mathrm{~min}, 4^{\circ} \mathrm{C}$ ) and was stored in aliquots at $-60^{\circ} \mathrm{C}$ until use.

\section{Isolated plasma $\mathrm{hBChE}$}

Isolated human butyrylcholinesterase (hBChE; E.C. 3.1.1.8) was obtained from Sigma. This enzyme was used for kinetic assays. 


\section{Production of recombinant $\mathrm{hBChE}$}

Recombinant hBChE was produced in Chinese hamster ovary (CHO) cells and secreted into serum-free culture medium [12]. Briefly, recombinant hBChE was a truncated monomer containing residues 1-529, whereas the tetramerization domain at the C-terminus was deleted. The glycan chain number was reduced by site-directed mutagenesis from 9 to 6 . Enzyme purification was implemented by affinity and ion exchange chromatography as described. The active-site concentration of highly purified enzyme was determined using diisopropyl fluorophosphate as the titrant as described [13]. This enzyme was used for crystallographic assays and for testing of enantioselectivity.

\section{Enzyme assays}

$h \mathrm{BChE}$ activities were measured spectrophotometrically (Cary 3Bio, Varian, Darmstadt) at $436 \mathrm{~nm}$ with a modified Ellman assay at $37^{\circ} \mathrm{C}[14,15]$. Though, regular Ellman assay at 412 $\mathrm{nm}$ works as well. The assay mixture $(3.16 \mathrm{ml})$ contained $1 \mathrm{mM}$ BTCh as substrate and 0.3 mM DTNB as chromogen in 0.1 M phosphate buffer ( $\mathrm{pH} 7.4)$.

\section{Determination of inhibition rate constants $\left(k_{i}\right)$}

The inhibition rate constants were determined at pseudo-first-order reaction conditions as described before [6]. In brief, appropriately diluted OP was added to temperature-equilibrated $\left(37^{\circ} \mathrm{C}\right)$ isolated $\mathrm{hBChE}$ at $\mathrm{t}=0$, an aliquot was removed after specified time intervals $(1-9$ min) and transferred to a cuvette for the determination of residual hBChE activity. $\mathrm{k}_{\mathrm{i}}$ was calculated from equation 1 :

$$
k_{i}=\frac{1}{[O P] * t} * \ln \frac{v_{0}}{v_{t}} \quad \text { eq. } 1
$$

where $[\mathrm{OP}]$ is the initial concentration of the tested $\mathrm{OP}, \mathrm{v}_{0}$ and $\mathrm{v}_{\mathrm{t}}$ are the reaction rates at time zero and at time $t$, respectively. The concentration ranges of OP used in the experiment were: tabun 20-80 nM; TA1 100-250 nM; TA1' 1200-1800 nM; TA2 200-500 nM; TA3 20-50 $\mu$ M; TA4 50-125 nM; TA5 70-100 nM; TA6 50-90 nM.

\section{Test of enantioselectivity for TA 4-6}

Determination of inhibition enantioselectivity of recombinant $\mathrm{hBChE}$ was performed under second-order conditions as described [11]. In brief, incubation was performed at $25{ }^{\circ} \mathrm{C}$ in 50 $\mathrm{mM}$ sodium phosphate buffer $\mathrm{pH} 7.0$ containing 5\% isopropanol, $1 \mathrm{mg} / \mathrm{mL}$ BSA, a 
concentration of $\mathrm{hBChE}$ about $30 \mathrm{nM}$ while that of tabun analogues ranged between 15 and $200 \mathrm{nM}$. Residual activity was monitored at $25^{\circ} \mathrm{C}$ and $\mathrm{pH} 7.0$, according to Ellman's method using $1 \mathrm{mM}$ BTCh and $0.5 \mathrm{mM}$ DTNB. Under second order conditions, the concentration of residual active enzyme follows equation 2 , as a function of time [11] :

eq. 2

$$
E=E_{0} \frac{E_{0}-\frac{\left[O P_{0}\right]}{n}}{E_{0}-\frac{\left[O P_{0}\right]}{n} e^{\left(\frac{\left[O P_{0}\right]}{n}-E_{0}\right) k i^{\prime} \cdot t}}
$$

Where $\mathrm{E}$ is the concentration of residual active enzyme at time $=t, E_{0}$ the initial concentration of active enzyme, $\left[\mathrm{OP}_{0}\right]$ the initial concentration of racemic $\mathrm{OP}, \mathrm{n}$ the number of enantiomers, and ki' the bimolecular rate constant of the most active enantiomer assuming the inhibition rate of the other enantiomer is negligible. Analysis of the kinetic data was performed using GOSA-fit, a fitting software based on a simulated annealing algorithm (BioLog, Toulouse, France; http://www.bio-log.biz), allowing simultaneous fitting of all the data at once.

\section{Oxime screening for the reactivation of OP-inhibited hBChE}

Human plasma was incubated with a small volume $(1 \% \mathrm{v} / \mathrm{v})$ of appropriate concentrations of tabun and compounds TA $1-6,30$ min at $37^{\circ} \mathrm{C}$ to achieve an $\mathrm{hBChE}$ inhibition of $>90 \%$. Thereafter, the treated samples were dialyzed (phosphate buffer, $0.1 \mathrm{M}, \mathrm{pH} 7.4$ ) overnight at $4^{\circ} \mathrm{C}$ to remove residual inhibitor. The inhibitory activity of the samples was tested by $30 \mathrm{~min}$ of incubation at $37^{\circ} \mathrm{C}$ of treated plasma and control $\mathrm{hBChE}$ in order to verify the absence of residual inhibitor. OP-inhibited $\mathrm{hBChE}$ was incubated at $37^{\circ} \mathrm{C}$ with $1 \mathrm{mM}$ obidoxime, TMB4, HI-6 or HLö 7 and the hBChE activity was measured at specified time intervals $(1-120$ min). Enzyme activities were referred to control activity and the \% reactivation was calculated according to de Jong and Wolring [16]. Experiments were performed in duplicate.

\section{Kinetics of obidoxime reactivation}

Due to low reactivating potency, the reactivation kinetics were determined at $37^{\circ} \mathrm{C}$ by a discontinuous procedure as described before $[6,17]$ which allowed the use of high obidoxime concentrations in incubation mixture (up to $5 \mathrm{mM}$ ). OP-inhibited hBChE was incubated with different obidoxime concentrations and $1 \mathrm{mM}$ BTCh. Aliquots were transferred to cuvettes after specified time intervals $(2-45 \mathrm{~min})$ for measurement of $\mathrm{hBChE}$ activity. The 
dissociation constant $\mathrm{K}_{\mathrm{D}}$ of enzyme-obidoxime complex, the reactivity rate constant $\mathrm{k}_{\mathrm{r}}$ and the second order reactivation rate constant $\mathrm{k}_{\mathrm{r} 2}$ were calculated by standard procedure [6]. Eight to ten obidoxime concentrations, $100-5000 \mu \mathrm{M}$ with tabun- and TA3-inhibited BChE and $50-3000 \mu \mathrm{M}$ with TA4 - TA6-inhibited enzyme, were used for the determination of reactivation rate constants.

\section{Determination of rate constants for aging $\left(k_{a}\right)$ and spontaneous reactivation $\left(k_{s}\right)$}

OP-inhibited $\mathrm{hBChE}$ was brought to $37^{\circ} \mathrm{C}(\mathrm{t}=0)$. Then, aliquots were taken after various time intervals for determination of $\mathrm{BChE}$ activity (spontaneous reactivation) and of the decrease of oxime-induced reactivation (aging). Hereby, small aliquots were incubated $1 \mathrm{~h}$ with $1 \mathrm{mM}$ obidoxime. Data from duplicate experiments were referred to control activities and the percentage reactivation (\% react) was calculated. The pseudo first-order rate constants $k_{s}$ (spontaneous reactivation) and $\mathrm{k}_{\mathrm{a}}$ (aging) were calculated by a non-linear regression model $[6]$.

\section{Crystallization of nonaged TA1, TA4, TA5 and TA6-inhibited hBChE}

The mother liquor was $0.1 \mathrm{M}$ MES buffer, pH 6.5, with $2.1 \mathrm{M}$ ammonium sulfate. Stock solutions of tabun analogues were $10 \mathrm{mM}$ in isopropanol. Native enzyme was crystallized using the hanging drop method [12]. Crystals grew in one week at $20{ }^{\circ} \mathrm{C}$. Then, the OPhBChE conjugates were prepared by soaking crystals for 10 min in $0.1 \mathrm{M}$ MES buffer, $\mathrm{pH}$ 6.5, with $2.1 \mathrm{M}$ ammonium sulfate, containing $2 \mathrm{mM}$ tabun analogue. The crystals were washed with a cryoprotectant solution [0.1 M MES buffer with 2.1 M ammonium sulfate, containing $20 \%$ glycerol], and then flash-cooled in liquid nitrogen.

\section{Crystallization of aged TA1, TA4 and TA5-hBChE}

Purified enzyme ( $9 \mathrm{mg} / \mathrm{mL}$ ) was inhibited by $2 \mathrm{mM}$ of tabun analogue compounds, in $10 \mathrm{mM}$ Tris $\mathrm{HCl}$ buffer, $\mathrm{pH}$ 7.4. The inhibited enzyme was crystallized using the hanging drop method [12]. Hereby, crystals grew in one week at $20{ }^{\circ} \mathrm{C}$. The time between phosphylation and data collection was sufficiently long ( $>2$ week) to achieve completion of the aging reaction (aging half time $<70 \mathrm{~h}$ ).

\section{X-ray Data Collection and Structure Solution of hBChE}

Diffraction data were collected at $\lambda=0.932 \AA$ wavelength at the ID14-eh2 beamline of the European Synchrotron Radiation Facility (ESRF, Grenoble) with a MAR-Research CCD 
detector. All data sets were processed with XDS and the structures were solved by use of the CCP4 suite [18]. An initial solution model was determined by molecular replacement with MolRep [19], starting with the recombinant hBChE structure (PDB entry 1P0I) from which all ligands (butyrate, glycerol, ions) and glycan chains were removed. For all diffraction data sets, the model was refined as follows: an initial rigid-body refinement with REFMAC5 [20] was followed by iterative cycles of model building with Coot [21], and finally restrained refinement was carried out with REFMAC5 and Phenix [22]. The covalently bound inhibitors and their descriptions were built using the Dundee PRODRG2.5 server including energy minimization using GROMOS96.1 forcefield. 


\section{Results}

\section{Inhibition of hBChE by tabun analogues}

The bimolecular rate constants, $\mathrm{k}_{\mathrm{i}}$, for inhibition of human $\mathrm{BChE}$ by tabun and its analogues (TA1 to TA6) in the absence of substrate are in table 1. Phosphoramidocyanidates react faster than homologous phosphoramidofluoridate: TA1 with cyanide as leaving group reacts 17 times faster than TA1' with fluoride as leaving group. Hereby, the inhibitory potency was related to the chain length of the $\mathrm{N}$-alkyl group. With tabun and its closest analogues, TA1 and TA2, bearing a cyanide leaving group and dialkyl chains, $\mathrm{k}_{\mathrm{i}}$ values decreased with increasing chain length. In contrast, for TA 4, 5 and 6, bearing a mono-N-alkyl group, the inhibitory potency increases with chain length, each carbon addition doubling the bimolecular rate constant.

\section{Determination of enantioselectivity}

The time dependence of the inhibition of $\mathrm{hBChE}$ by TA4, TA5 and TA6, under second-order conditions, is shown in figure 3. Fitting the data to equation 2 yields ki' $=2.4 \pm 0.210^{6} \mathrm{M}^{-}$ ${ }^{1} \cdot \mathrm{min}^{-1}$ and $\mathrm{n}=1.8 \pm 0.1$ for TA4, $\mathrm{ki}{ }^{\prime}=8.4 \pm 0.510^{6} \mathrm{M}^{-1} \cdot \min ^{-1}$ and $\mathrm{n}=2.0 \pm 0.1$ for TA5, and $\mathrm{ki}{ }^{\prime}=$ $24 \pm 210^{6} \mathrm{M}^{-1} \cdot \mathrm{min}^{-1}$ and $\mathrm{n}=2.0 \pm 0.1$ for TA6. It follows that $\mathrm{hBChE}$ reacts preferentially with one enantiomer of tabun analogues. A similar enantiomeric preference was observed for inhibition of $\mathrm{hBChE}$ by tabun [11]. In this regard, ki differs from ki' because it is determined under pseudo first order conditions and describe the reaction of both enantiomers. Moreover, experimental conditions concerning temperature, $\mathrm{pH}$ and isopropanol-concentration were different.

\section{Reactivation of phosphoramidate-inhibited hBChE by oximes}

The reactivation of $\mathrm{OP}$-inhibited $\mathrm{hBChE}$ by oximes was tested with $1 \mathrm{mM}$ obidoxime, TMB4, HI-6 and HLö 7 (Fig. 4). Tabun-inhibited hBChE could be partially reactivated by oximes, the extent of reactivation decreased in the order obidoxime $>$ TMB-4 $>$ HLö $7>$ HI-6. Oximeinduced reactivation was rather ineffective with $\mathrm{hBChE}$ inhibited by $\mathrm{TA} 1$ and TA2. Interestingly, reactivation of $\mathrm{hBChE}$ inhibited by $\mathrm{TA} 3$ followed a different pattern. All oximes were capable of reactivating the inhibited enzyme, and after $120 \mathrm{~min}$ incubation the recovery in $\mathrm{hBChE}$ activity was in the range of $60-85 \%$. 
In contrast all oximes reactivated $\mathrm{hBChE}$ inhibited by $\mathrm{N}$-monoalkyl tabun analogues, i.e. TA4, TA5 and TA6 completely, although there were some differences between oximes regarding velocity and extent of reactivation (Fig. 4).

The reactivation kinetics of $\mathrm{hBChE}$ inhibited by tabun and its analogues TA3 to TA6, was determined with obidoxime. The reactivation constants are in table $1 . \mathrm{K}_{\mathrm{D}}$ is the dissociation constant of oxime-OP-hBChE complex, $\mathrm{kr}$ the reactivation constant and $\mathrm{kr} 2$ describes the specific reactivity. No rate constants could be determined with hBChE inhibited by TA1 and TA2, due to low reactivation extent. TA3-inhibited hBChE was, compared to tabun, substantially more susceptible towards reactivation.

Reactivation of hBChE inhibited by N-monoalkyl tabun analogues, TA4, TA5 and TA6, resulted in comparable second-order rate constant $\mathrm{k}_{\mathrm{r} 2}$ (table 1). There was only little difference in $K_{D}$ and $k_{r}$ between TA5 and TA6 while both values were substantially lower with TA4.

\section{Aging and spontaneous reactivation of phosphoramidate-inhibited hBChE}

Aging and spontaneous reactivation constants are in table 1. Aging kinetics was not determined for TA1 and TA2 due to insufficient reactivation extent by oximes. Tabun and its $\mathrm{N}, \mathrm{N}-$ di-i-propyl analogue (TA3) showed comparable aging kinetics with a $\mathrm{k}_{\mathrm{a}}$ of $0.1 \mathrm{~h}^{-1}$ and $0.094 \mathrm{~h}^{-1}$, respectively. hBChE inhibited by $\mathrm{N}$-monoalkyl tabun analogues TA4, 5 and 6, was susceptible towards aging and spontaneous reactivation. Hereby, the velocity of spontaneous reactivation increased with the chain length of the $\mathrm{N}$-alkyl group, whereas the aging rates are comparable and noticeably slower than for tabun-inhibited hBChE (table 1).

\section{$\mathrm{X}$-ray structure of non-aged tabun analogue-hBChE conjugates}

Data were collected from tetragonal crystals of space group $I 422$ and refined to $2.3 \AA, 2.15 \AA$, $2.1 \AA$ and $2.1 \AA$ for TA1, TA4, TA5 and TA6, respectively. Corresponding data and refinement statistics are shown in table $2 \mathrm{~A}$. A strong peak of positive electron density (10-15 $\sigma)$ within covalent bond distance of the catalytic serine was observed in the initial $\mid$ Fo $|-| F c \mid$ map for each conjugates. This confirms that inhibitors were bound to the active site serine after 5-min soaking. The refinement yields an adduct that exhibits $\mathrm{P}(\mathrm{R})$ stereochemistry for the chiral phosphorus atom except for TA4 that exhibits $\mathrm{P}(\mathrm{S})$ stereochemistry (Fig. 5). Given the good resolution $(2.15 \AA)$ and the map quality there is no ambiguity in TA4 stereochemistry assignment. As a control, we also placed the presumably incorrect stereoisomer of TA4 and performed one round of structural refinement. Distinct positive and 
negative difference density ( $|\mathrm{Fo}|-|\mathrm{Fc}|$ map) peaks support the conclusion that the adduct intermediate observed in the structures is in the $\mathrm{S}$ conformation. There was no significant movement of residue side-chains compared to the native enzyme (pdb code: 1P0I).

There is a strong peak of positive electron density in the initial $|\mathrm{Fo}|-|\mathrm{Fc}|$ map close to Trp82. This density could not be reasonably modeled. It is most likely due to the presence of the small ligand that was previously observed in other structures of $\operatorname{hBChE}[23,24]$. This unknown ligand appears stacked against $\operatorname{Trp} 82$ and interacts with water molecules. For TA1 there is no large blob of density, but electron density corresponding to two water molecules. The phosphorus atoms were found at covalent bonding distance from the Ser1980 $\gamma$ atoms (about $1.6 \AA$ ) and the P-N distance were refined to values ranging from $1.6 \AA$ to $1.7 \AA . \mathrm{O} 2$ of the phosphoramidate moiety is at hydrogen bonding distance of the main chain amide nitrogen of residues forming the oxyanion hole (Ala199/Gly116/Gly117). The N-alkyl subsituents were located in the acyl-binding pocket with the alkyl groups pointing toward the top of the gorge, except for TA4. For this later, the N-alkyl group projects towards the choline binding pocket and the ethoxy moiety, located in the acyl-binding pocket, is weakly interacting with Leu286 and Phe398. On the choline binding pocket side, ethoxy oxygen O2 of TA1, TA5, TA6 and N of TA4 are at hydrogen bonding distance of His438Nع2.

For TA1, N-ethyl group makes van der Waals contact with Trp231 and Phe329. N-diethyl substituent of TA1 imposes much more stress on the residue of the acyl-binding pocket such that we observed a concerted shift about $0.5 \AA$ of Phe398, Phe329 and Leu286.

\section{X-ray structure of aged tabun analogue-hBChE conjugates}

The structures of aged forms were obtained by crystallization of inhibited enzyme in solution. X-ray crystallographic data were collected from tetragonal crystals of space group I422, except for TA5 (P4212) and refined to $2.25 \AA, 2.0 \AA$ and $3.1 \AA$ for TA1, TA4 and TA5, respectively. Corresponding data and refinement statistics are shown in table $2 \mathrm{~B}$. The orientation of aged conjugates is identical to the non-aged conjugates and confirms that the stereoselectivity of the inhibition is identical in cristallo and in solution (Fig. 5). This also confirms the initial stereochemistry of TA4. As expected in aged forms, the alkyl group projecting toward the choline-binding pocket is missing. This shows that TA1 and TA5 undergo classical O-dealkylation as previously observed for tabun [11], phosphates and phosphonates [8]. On the other hand, TA4 likely undergoes deamination. As a result of aging, O3 forms a salt bridge with protonated His438Nع2 (2.7-2.8 $\AA$ ). For each conjugates, the 
phosphoramidyl moiety has tilted toward His438 as a consequence of the salt bridge formation. For TA1, this also translates into a release of stress in the acyl-binding pocket so that only Phe329 remains shifted. For TA4 and TA5, van der Waals contacts with Trp231 and Phe329 are similar as in their nonaged form. The small ligand is still present stacked to Trp82 and interacts with $\mathrm{O} 3$ of the aged adducts of TA1, TA4 and is replace by two water molecules in structure of aged TA5-hBChE conjugate.

\section{Discussion}

The higher inhibitory potency of phosphoramidocyanidates compared to homologous phosphoramidofluoridates (table 1) could be related to the electron-withdrawing effect of cyanide that is stronger than that of fluorine. Cyanide is also a mesomer acceptor in contrast to fluorine. It follows that the phosphorus atom of phosphoramidocyanidates is more electrophilic and more reactive. It is also possible that during the approach of the inhibitor, the cyanide substituent makes more favorable interactions with the enzyme than fluorine.

Non-aged and aged structures were obtained for tabun analogues TA1, TA4, TA5 and nonaged structures for TA6 (Fig. 5). We failed to obtain conjugates with TA3 and TA2, most likely because of their poor reactivity with $\mathrm{hBChE}$ in relation to their large size. The X-Ray structures and second order kinetic data show that there is enantioselectivity for reaction with fluorophosphoramidate analogues (Fig. 3, Fig. 5).

We observed that the N,N-dialkyl substituent of TA1 is located in the acyl-binding pocket, similarly to tabun [11]. The bulky N,N-diethyl substituent of TA1 fits in the acyl-binding pocket, only inducing slight rotation of Leu286, and the phosphoramidate head is pushed toward the choline-binding pocket compared to TA5 and TA6 (Fig. 6). The steric hindrance resulting from N,N-diethyl group, forces the O-ethoxy substituent to orient toward the bottom of the choline-binding pocket. This notably prevents the binding of the unknown ligand, just leaving enough room for two water molecules (Fig. 5, Fig. 6). As for tabun, the orientation of TA1 is explained by delocalization of the N-lone pair and subsequent cation- $\pi$ interactions between the diethylamino and Trp231.

For N-monoalkyl analogues (TA4-6), the orientation of the OP follows different rules. The larger substituent, wherever the ethoxy or amino group, is located in the acyl-binding pocket, forming more van der Waals interactions than the smaller substituent and suggesting that cation- $\pi$ interactions of N-monoalkyl group and Trp231 is secondary in importance. Indeed, we expect weaker cation- $\pi$ interactions because delocalization of the nitrogen lone pair is less 
stabilized for primary amines compared to secondary amines, thus leading to a lesser positive partial charge on the amino group. For the N-monoalkyl analogues, the length of the substituent appears to prime on the interaction of $\mathrm{N}$-methyl with the acyl-binding pocket. Actually, this could result from preference of hBChE's acyl-binding pocket for larger than 2atoms linear substituents. Indeed, when the substituent is shorter, for example a single atom as for soman-aged $\mathrm{hBChE}$ conjugate (pdb code : 1p0q), it induces a rearrangement of the acylloop (Val279-Asn289). Thus, for TA4 the O-ethyl (3-atom chain) substituent is located in the acyl-binding pocket and the shorter $\mathrm{N}$-methyl (2-atom chain) toward the choline-binding pocket (Fig. 5).

For TA5, we cannot conclude regarding the orientation, as both substituents are undistinguishable by X-ray crystallography. However, second order kinetics shows that $\mathrm{hBChE}$ reacts specifically with one enantiomer of TA5. Since van der Waals interactions should be equivalent for both substituents, we suppose that the ethylamino group interacts preferably with Trp231 through cation- $\pi$ interactions, whereas the ethoxy oxygen interacts with His438Ne2.

Crystallographic data show that aging occurs through classical O-dealkylation for TA1, TA6 (Fig. 5), similarly to previous observation made on tabun-ChEs conjugates [11]. Unexpectedly, there was also aging for TA4 although the ethoxy group is located in the acylbinding pocket (Fig. 5). These results show that aging of phosphoramidates systematically involves departure of the substituent in the choline-binding pocket. The mechanism differs, depending on the nature of the substituent O-alkyl or N-alkyl group (Fig. 7). When this substituent is the ethoxy, aging proceeds through dealkylation: His438 imidazolium stabilizes a developing negative charge on the $\mathrm{C}-\mathrm{O}^{\delta-}$ oxygen, and a water molecule activated by Glu197 attacks the carbocationic center that appears on the $\mathrm{C}^{\delta+}-\mathrm{O}$ carbon. This leads to scission of the $\mathrm{C}-\mathrm{O}$ bond, release of the ethoxy moiety, and subsequent formation of the salt bridge between the phosphorous O3-oxygen and the imidazolium. This is illustrated with TA1, TA6 and previous solved structures of aged $\mathrm{hBChE}$ inhibited by tabun, soman, DFP, echothiophate [11, $23,24]$.

For TA4, the N-methyl substituent projects towards the choline-binding site so that aging proceeds through deamination: His438 imidazolium protonates the methylamine group. Extremely rapid deamination after nucleophilic attack by water can be assumed since a protonated methylamine is an excellent leaving group. This mechanism, like dealkylation, leads to the formation of a salt bridge with a short interatomic distance, as shown by the 
crystal structure $(\mathrm{His} 438 \mathrm{~N} \varepsilon 2$ and $\mathrm{O} 3=2.7 \AA)$. Remarkably, aging rates of all mono-alkyl analogues are comparable despite the difference of mechanism.

$\mathrm{N}$,N-dialkyl compounds are generally less potent inhibitors of hBChE than N-monoalkyl analogues (table 1). N,N-dialkyl compounds are less reactive because steric hindrance from the two alkyl side-chains prevents a correct approach of the OP to the catalytic serine. Moreover, the two alkyls chains have electron-releasing effect that favors delocalization of the nitrogen lone pair on the phosphorous atom, thus decreasing the electrophilicity of this later. Additionally, Trp231 could stabilize positive partial charges on the amino group when the dialkylamino substituent is in the acyl-binding pocket, thus further favoring delocalization of the N-lone pair.

The remarkable correlation between the inhibitory potency and the N-monoalkyl chain length is most likely related to increasingly optimized interactions with the acyl-binding pocket as shown by the X-ray structures (Fig.5).

The electronic and steric effects have similar influence for the reactivation reaction by oximes of N,N-dialkyl compared to N-monoalkyl compounds (Fig. 4). In addition to the poorer reactivity of the phosphorus atom, the two alkyl side-chains prevents a correct approach of the oxime in apical position, as illustrated in figure 6.

Obidoxime, TMB-4, HLö 7, HI-6 have a similar efficiency on each phosphoramidyl-hBChE conjugates. The increase in chain length of N-monoalkyl compounds does not affect the affinity of oximes to hBChE conjugates by contrast to hAChE [7], because the long chain can easily fit in the acyl-binding pocket.

These bispyridinium oximes are much less efficient than for phosphoramidyl-hAChE [7], despite Tyr124, that hinders the phosphorus atom of hAChE conjugates for an optimal apical approach, is not conserved in hBChE (Gln119). This is due to a lower affinity of oximes for phosphoramidyl-hBChE conjugates. Indeed, these bispyridinium oximes bind to the peripheral site of $\mathrm{hAChE}$, through aromatic stacking and cation- $\pi$ interactions between one pyridinium moiety and peripheral site residues (Trp286, Tyr124 and Tyr72) [25]. In hBChE, these aromatic residues are not conserved and bispyridinium oximes cannot interact in the same fashion. Considering that the OPs occupy the bottom of the active site gorge, and that the nucleophile function must be in apical position for optimal in-line attack, efficient reactivators must bind to the gorge entrance of $\mathrm{hBChE}$. Efforts should be pursue to design such reactivators. 
In summary, this work provides a better understanding of inhibition of $\mathrm{hBChE}$ by phosphoramidates and aging of the conjugates, and provides a structural basis for rational design by molecular modeling of hBChE-based catalytic bioscavengers and oximes able to reactivate phosphoramidyl-hBChE. Great improvement in prophylaxis, decontamination and treatment of organophosphate poisoning are expected in the near future as $\mathrm{hBChE}$ is close to FDA approval.

\section{Acknowledgments}

This work was supported by DGA grant 03co10-05/PEA 01087 to PM, DGA/PEA 08co501 and ANR-06-BLAN-0163 to FN. The authors are grateful to T. Hamig and L. Windisch for their skilful and engaged technical assistance. 


\section{References}

1 Lockridge, O. and Masson, P. (2000) Pesticides and susceptible populations: people with butyrylcholinesterase genetic variants may be at risk. Neurotoxicology. 21, 113126

2 Masson, P., Carletti, E. and Nachon, F. (2008) Structure, activities and biomedical applications of human butyrylcholinesterase. Pep. Prot. Letters. in press

3 Eddleston, M. (2008) The pathophysiology of organophosphorus pesticide selfpoisoning is not so simple. Neth. J. Med. 66, 146-148

4 Konradsen, F., Dawson, A. H., Eddleston, M. and Gunnell, D. (2007) Pesticide selfpoisoning: thinking outside the box. Lancet. 369, 169-170

5 Saxena, A., Redman, A. M., Jiang, X., Lockridge, O. and Doctor, B. P. (1997) Differences in active site gorge dimensions of cholinesterases revealed by binding of inhibitors to human butyrylcholinesterase. Biochemistry. 36, 14642-14651

6 Worek, F., Thiermann, H., Szinicz, L. and Eyer, P. (2004) Kinetic analysis of interactions between human acetylcholinesterase, structurally different organophosphorus compounds and oximes. Biochem. Pharmacol. 68, 2237-2248

7 Worek, F., Aurbek, N., Koller, M., Becker, C., Eyer, P. and Thiermann, H. (2007) Kinetic analysis of reactivation and aging of human acetylcholinesterase inhibited by different phosphoramidates. Biochem. Pharmacol. 73, 1807-1817

8 Li, H., Schopfer, L. M., Nachon, F., Froment, M. T., Masson, P. and Lockridge, O. (2007) Aging pathways for organophosphate-inhibited human butyrylcholinesterase, including novel pathways for isomalathion, resolved by mass spectrometry. Toxicol. Sci. 100, 136-145

9 Shafferman, A., Ordentlich, A., Barak, D., Stein, D., Ariel, N. and Velan, B. (1997) Aging of somanyl-acetylcholinesterase adducts: facts and models. Biochem. J. 324 ( Pt 3), 996-998

10 Kovach, I. M., Akhmetshin, R., Enyedy, I. J. and Viragh, C. (1997) A self-consistent mechanism for dealkylation in soman-inhibited acetylcholinesterase. Biochem. J. 324 ( Pt 3), 995-996

11 Carletti, E., Li, H., Li, B., Ekström, F., Nicolet, Y., Loiodice, M., Gillon, E., Froment, M. T., Lockridge, O., Schopfer, L. M., Masson, P. and Nachon, F. (2008) Aging of cholinesterases phosphylated by tabun proceeds through O-dealkylation. J. Am. Chem. Soc. 130, 16011-16020

12 Nachon, F., Nicolet, Y., Viguie, N., Masson, P., Fontecilla-Camps, J. C. and Lockridge, O. (2002) Engineering of a monomeric and low-glycosylated form of human butyrylcholinesterase: expression, purification, characterization and crystallization. Eur. J. Biochem. 269, 630-637

13 Amitai, G., Moorad, D., Adani, R. and Doctor, B. P. (1998) Inhibition of acetylcholinesterase and butyrylcholinesterase by chlorpyrifos-oxon. Biochem. Pharmacol. 56, 293-299

14 Worek, F., Mast, U., Kiderlen, D., Diepold, C. and Eyer, P. (1999) Improved determination of acetylcholinesterase activity in human whole blood. Clin. Chim. Acta. 288, 73-90

15 Eyer, P., Worek, F., Kiderlen, D., Sinko, G., Stuglin, A., Simeon-Rudolf, V. and Reiner, E. (2003) Molar absorption coefficients for the reduced Ellman reagent: reassessment. Anal. Biochem. 312, 224-227

16 de Jong, L. P. and Wolring, G. Z. (1978) Effect of 1-(AR)alkyl-2-hydroxyiminomethylpyridinium salts on reactivation and aging of acetyl-cholinesterase inhibited by ethyl dimethylphosphoramidocyanidate (tabun). Biochem. Pharmacol. 27, 2229-2235

17 Wang, R. I. and Henschel, E. O. (1967) Semiquantitative determination of cholinesterase activity in human plasma: test-paper method. Anesth. Analg. 46, 281285 
18 Collaborative-Computational-Project4. (1994) The CCP4 suite: programs for protein crystallography. Acta. Crystallogr. D. Biol. Crystallogr. 50, 760-763

19 Vagin, A. and Teplyakov, A. (1997) MOLREP: an automatedprogram for molecular replacement. J. Appl. Crystallogr. 30, 1022-1025

20 Murshudov, G. N., Vagin, A. A. and Dodson, E. J. (1997) Refinement of macromolecular structures by the maximum-likelihood method. Acta. Crystallogr. D. Biol. Crystallogr. 53, 240-255

21 Emsley, P. and Cowtan, K. (2004) Coot: model-building tools for molecular graphics. Acta. Crystallogr. D. Biol. Crystallogr. 60, 2126-2132

22 Adams, P. D., Grosse-Kunstleve, R. W., Hung, L. W., Ioerger, T. R., McCoy, A. J., Moriarty, N. W., Read, R. J., Sacchettini, J. C., Sauter, N. K. and Terwilliger, T. C. (2002) PHENIX: building new software for automated crystallographic structure determination. Acta. Crystallogr. D. Biol. Crystallogr. 58, 1948-1954

23 Nachon, F., Asojo, O. A., Borgstahl, G. E., Masson, P. and Lockridge, O. (2005) Role of water in aging of human butyrylcholinesterase inhibited by echothiophate: the crystal structure suggests two alternative mechanisms of aging. Biochemistry. 44, 1154-1162

24 Nicolet, Y., Lockridge, O., Masson, P., Fontecilla-Camps, J. C. and Nachon, F. (2003) Crystal structure of human butyrylcholinesterase and of its complexes with substrate and products. J. Biol. Chem. 278, 41141-41147

25 Ekstrom, F. J., Astot, C. and Pang, Y. P. (2007) Novel nerve-agent antidote design based on crystallographic and mass spectrometric analyses of tabun-conjugated acetylcholinesterase in complex with antidotes. Clin. Pharmacol. Ther. 82, 282-293 


\section{Tables}

\begin{tabular}{|c|c|c|c|c|c|c|c|}
\hline $\bar{x}$ & $\mathrm{~N}$-group & $\begin{array}{c}k_{i}\left(10^{6}\right) \\
\left(M^{-1} \min ^{-1}\right)\end{array}$ & $K_{D}(\mu M)$ & $k_{r}\left(\min ^{-1}\right)$ & $\begin{array}{c}\mathbf{k}_{\mathrm{r} 2} \\
\left(\mathrm{mM}^{-1} \mathrm{~min}^{-1}\right)\end{array}$ & $k_{a}\left(h^{-1}\right)$ & $k_{s}\left(h^{-1}\right)$ \\
\hline tabun & & $30 \pm 8$ & $1470 \pm 250$ & $0.02 \pm 0.002$ & $0.017 \pm 0.006$ & 0.01 & $\varnothing^{\mathrm{c}}$ \\
\hline TA1 & & $1 \pm 0.0$ & $\varnothing^{a}$ & $\varnothing^{a}$ & $\varnothing^{a}$ & & $\varnothing^{c}$ \\
\hline TA1' & & $0.06 \pm 0.003$ & $\varnothing^{\mathrm{a}}$ & $\varnothing^{a}$ & $\varnothing^{a}$ & & $\varnothing^{c}$ \\
\hline TA2 & & $0.3 \pm 0.03$ & $\varnothing^{\mathrm{a}}$ & $\varnothing^{a}$ & $\varnothing^{a}$ & & $\varnothing^{\mathrm{c}}$ \\
\hline TA3 & & $0.012 \pm 0.001$ & $920^{d}$ & $0.09^{d}$ & 0 & $0.09 \pm 0.0001$ & $\varnothing^{c}$ \\
\hline TA4 & $\mathrm{HN}^{-}$ & $4 \pm 0.5$ & $370 \pm 40$ & $0.042 \pm 0.001$ & $0.117 \pm 0.010$ & $0.011 \pm 0.0003$ & $\begin{array}{l}0.004 \pm \\
0.0001\end{array}$ \\
\hline TA5 & $\mathrm{HN}^{-}$ & $8 \pm 0.6$ & $630 \pm 60$ & $0.072 \pm 0.003$ & $0.114 \pm 0.002$ & $0.012 \pm 0.0002$ & $\begin{array}{l}0.008 \pm \\
0.0001\end{array}$ \\
\hline TA6 & $\mathrm{HN}^{-}$ & $17 \pm 0.3$ & $660 \pm 50$ & $0.069 \pm 0.002$ & $0.108 \pm 0.002$ & $0.015 \pm 0.0005$ & $\begin{array}{l}0.019 \pm \\
0.0004\end{array}$ \\
\hline \multicolumn{8}{|c|}{$\begin{array}{l}\text { a Failure of oximes to reactivate inhibited } \mathrm{hBChE} \\
\mathrm{b} \text { Not feasible } \\
\text { d No spontaneous reactivation of inhibited } \mathrm{hBChE} \text { activity during the observation period } \\
\text { Single experiment }\end{array}$} \\
\hline
\end{tabular}




\begin{tabular}{|c|c|c|c|c|}
\hline \multicolumn{5}{|c|}{ Table 2A: Data Collection and Refinement Statistics } \\
\hline & TA1-hBChE & TA4-hBChE & TA5-hBChE & TA6-hBChE \\
\hline & \multicolumn{4}{|c|}{ (non-aged) } \\
\hline \multicolumn{5}{|l|}{ data } \\
\hline space group & $I 422$ & $I 422$ & $I 422$ & $I 422$ \\
\hline unit cell axes, $a=b, c(\AA)$ & $155.04,126.21$ & $155.01,126.84$ & $155.02,127.22$ & $155.24,127.25$ \\
\hline no. of reflections & 240729 & 302893 & 328846 & 314110 \\
\hline unique reflections & 34103 & 41918 & 45161 & 45258 \\
\hline resolution $(\AA)$ & $47.8-2.3(2.4-2.3)$ & $49.0-2.15(2.2-2.15)$ & $55.1-2.1(2.2-2.1)$ & $55.1-2.1(2.2-2.1)$ \\
\hline completeness (\%) & 99.3 & 99.7 & 99.9 & 99.6 \\
\hline$R \operatorname{merge}^{*}(\%)$ & $8.5(34.9)$ & $8.1(29.6)$ & $8.6(28.4)$ & $9.7(35.5)$ \\
\hline$I / o ́(\mathrm{I})$ & $23.1(5.3)$ & $25.8(4.3)$ & $24.7(4.4)$ & $16.6(3.4)$ \\
\hline Redundancy & $7.1(7.2)$ & $7.2(7.2)$ & $7.3(7.4)$ & $6.9(5.9)$ \\
\hline \multicolumn{5}{|l|}{ Refinement Statistics } \\
\hline$R$-factor ${ }^{\dagger}\left(R-\right.$ free $\left.^{\dagger}\right)$ & $18.9(25.5)$ & $18.9(25.3)$ & $20.0(25.3)$ & $21.3(27.6)$ \\
\hline \\
\hline protein & 4240 & 4227 & 4213 & 4208 \\
\hline solvent & 335 & 343 & 342 & 384 \\
\hline others & 167 & 163 & 162 & 164 \\
\hline mean $B$-factor $\left(\AA^{2}\right)$ & 37.6 & 35.3 & 35.9 & 37.4 \\
\hline \multicolumn{5}{|l|}{ RMS from ideality } \\
\hline bond length $(\AA)$ & 0.017 & 0.018 & 0.017 & 0.033 \\
\hline angles (deg) & 1.757 & 1.875 & 1.782 & 2.897 \\
\hline chiral $\left(\AA^{3}\right)$ & 0.128 & 0.114 & 0.121 & 0.229 \\
\hline \multicolumn{5}{|c|}{ 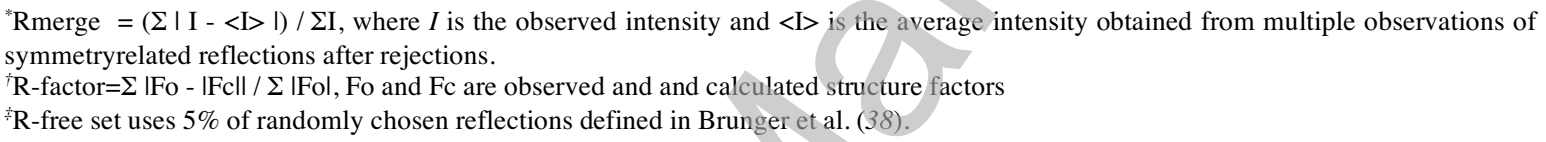 } \\
\hline
\end{tabular}

Table 2B: Data Collection and Refinement Statistics

\begin{tabular}{|c|c|c|c|}
\hline data & TA1-hBChE & $\begin{array}{c}\text { TA4-hBChE } \\
\text { (aged) }\end{array}$ & TA5-hBChE \\
\hline $\begin{array}{l}\text { space group } \\
\text { unit cell axes, } a=b, c(\AA) \\
\text { no. of reflections } \\
\text { unique reflections } \\
\text { resolution }(\AA) \\
\text { completeness }(\%) \\
R \text { merge* }(\%) \\
I / o ́(I) \\
\text { Redundancy }\end{array}$ & $\begin{array}{c}I 422 \\
155.04,126.21 \\
248419 \\
35245 \\
47.7-2.25(2.3-2.25) \\
96.3 \\
10.1(48.1) \\
15.3(4.0) \\
7.1(7.3) \\
\end{array}$ & $\begin{array}{c}I 422 \\
154.9,126.01 \\
391729 \\
51417 \\
48.9-2.0(2.1-2.0) \\
98.3 \\
8.8(37.5) \\
24.8(5.4) \\
7.6(7.4) \\
\end{array}$ & $\begin{array}{c}P 4212 \\
150.35,139.43 \\
167928 \\
28167 \\
48.4-3.1(3.2-3.1) \\
98.2 \\
10.1(30.5) \\
29.2(8.6) \\
5.7(5.9) \\
\end{array}$ \\
\hline Refinement Statistic & & & \\
\hline $\begin{array}{l}R \text {-factor }{ }^{\dagger}\left(R \text {-free }{ }^{\dagger}\right) \\
\text { no. of atoms } \\
\text { protein } \\
\text { solvent } \\
\text { others } \\
\text { mean } B \text {-factor }\left(\AA^{2}\right) \\
\text { RMS from ideality } \\
\text { bond length }(\AA) \\
\text { angles }(\text { deg }) \\
\text { chiral }\left(\AA^{3}\right)\end{array}$ & 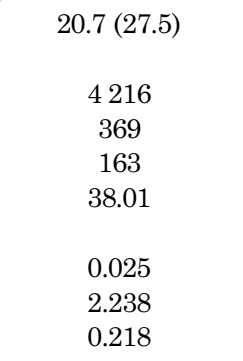 & 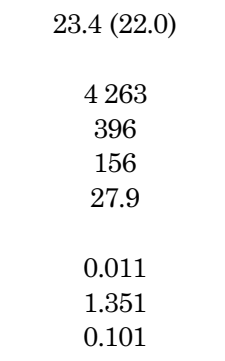 & $\begin{array}{c}22.0(25.3) \\
8396 \\
136 \\
268 \\
73.7 \\
\\
0.012 \\
1.415 \\
0.087\end{array}$ \\
\hline
\end{tabular}

${ }^{*}$ Rmerge $=(\Sigma \mid \mathrm{I}-\langle\mathrm{I}>\mathrm{I}) / \Sigma \mathrm{I}$, where $I$ is the observed intensity and $<\mathrm{I}>$ is the average intensity obtained from multiple observations of symmetryrelated reflections after rejections.

${ }^{\prime} \mathrm{R}$-factor $=\Sigma|\mathrm{Fo}-| \mathrm{Fc}|\mathrm{I} / \Sigma| \mathrm{Fol}, \mathrm{Fo}$ and $\mathrm{Fc}$ are observed and and calculated structure factors

${ }^{\mathrm{R}} \mathrm{R}$-free set uses $5 \%$ of randomly chosen reflections defined in Brunger et al. (38). 


\section{Figure legends}

Fig. 1 Chemical structures of tabun analogues (TA).

Fig. 2 Chemical structures of oximes used in this study.

Fig. 3 Irreversible inhibition $\mathrm{hBChE}$ by racemic tabun analogues performed under secondorder conditions. 29, 32.5 and $30 \mathrm{nM}$ of $\mathrm{hBChE}$ was inhibited by various concentrations of TA4, TA5 and TA6, respectively, and the residual enzyme activity was monitored as a function of time.

Fig. 4 Reactivation of OP-inhibited hBChE by obidoxime (black column), TMB-4 (dark grey column), HLö 7 (horizontally striped column) or HI-6 (light grey column). hBChE inhibited by tabun and its analogues (Fig. 1) was incubated with $1 \mathrm{mM}$ oxime and the $\mathrm{hBChE}$ activity was determined after $120 \mathrm{~min}$ incubation. Data are given as $\%$ reactivation (means $\pm \mathrm{SD}$ of 2 experiments).

Fig 5 Active site of non-aged and aged TA-hBChE conjugates. Key residues are represented as sticks with carbon in green for $\mathrm{OP}$ and blue for $\mathrm{hBChE}$ residues, nitrogen atoms in blue, oxygen atoms in red and phosphorus atom in orange. Water molecules are represented by red spheres and hydrogen bonds by red dashes. Electron density $2|\mathrm{Fo}|-|\mathrm{Fc}|$ is represented by a blue mesh, contoured at $1.0 \mathrm{\sigma}$ and $|F o|-|F c|$ is represented by green/red mesh contoured at $3.5 \sigma$.

Fig 6 Superimposition of the active site of non-aged form of TA1-hBChE (red), TA5hBChE (green) and TA6-hBChE (blue) conjugates.

Fig 7 Aging mechanisms of phosphoramidyl-hBChE conjugates. 
Fig. 1<smiles>CCOP(N)(=O)N(C)C</smiles><smiles>CCOP(N)(=O)N1C[C@@H]2CN(CC)[C@H]2C1</smiles>

tabun phosphoramidocyanidate (TA1)<smiles>CCOP(=O)(F)N(CC)CC</smiles>

Ethyl-N-diethyl fluorophosphate phosphoramin-propyl (TA1') (TA2)<smiles>CCOP(=O)(F)N(C(C)C)C(C)C</smiles><smiles></smiles>

Ethyl-N-di-isopropyl Ethyl-N-mono-methyl phosphorofluoridate phosphorofluoridate (TA3) (TA4)<smiles>CCNP(=O)(F)OCC</smiles><smiles>CCOPO</smiles>
$\mathrm{HN}$

Ethyl-N-mono-ethyl Ethyl-N-mono-n-propy! phosphorofluoridate phosphorofluoridate (TA5) (TA6) 
Fig. 2<smiles>O/N=C\c1cc[n+](CCC[n+]2ccc(/C=N\O)cc2)cc1</smiles>
obidoxime TMB-4<smiles>NC(=O)c1cc[n+](COC[n+]2ccccc2/C=N/O)cc1</smiles><smiles>NC(=O)c1cc[n+](CCC[n+]2ccc(/C=N/O)cc2/C=N/O)cc1</smiles> 
Fig. 3
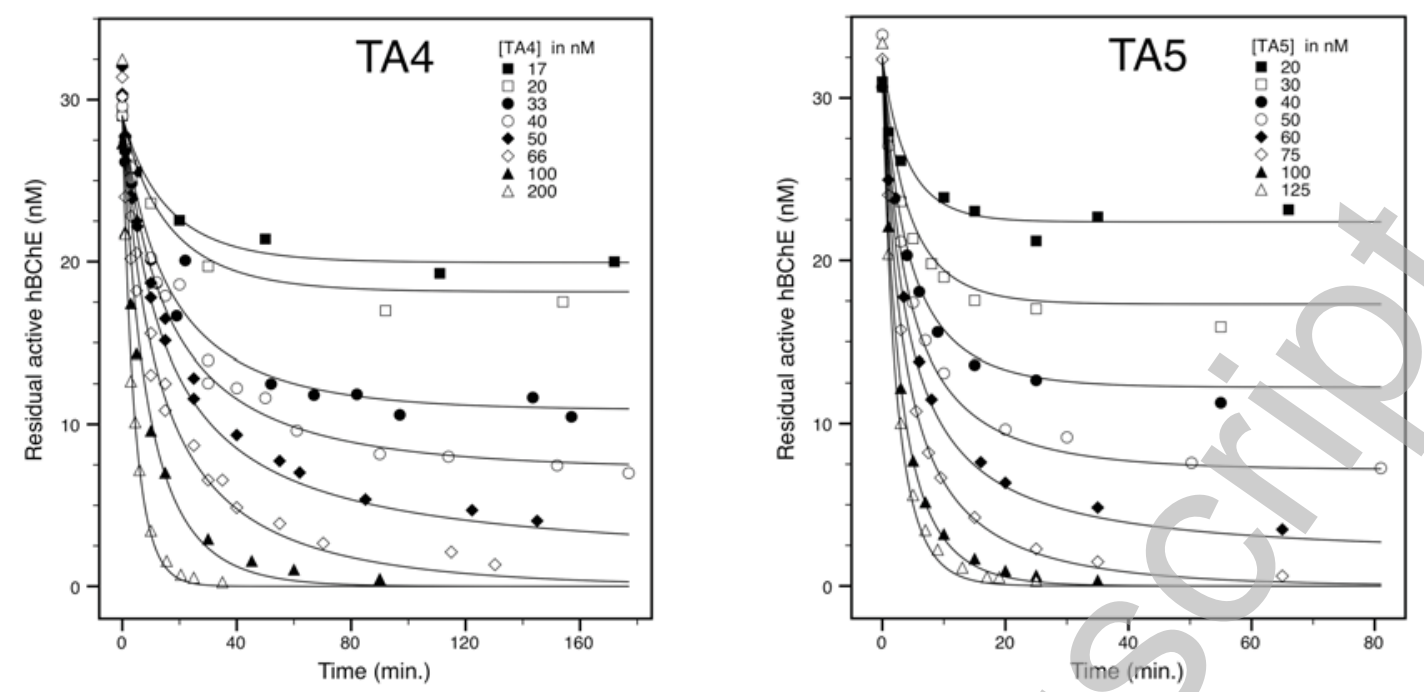

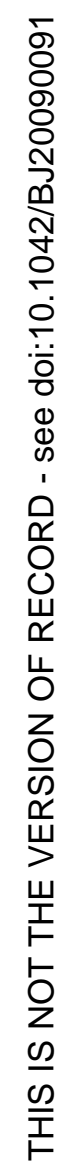

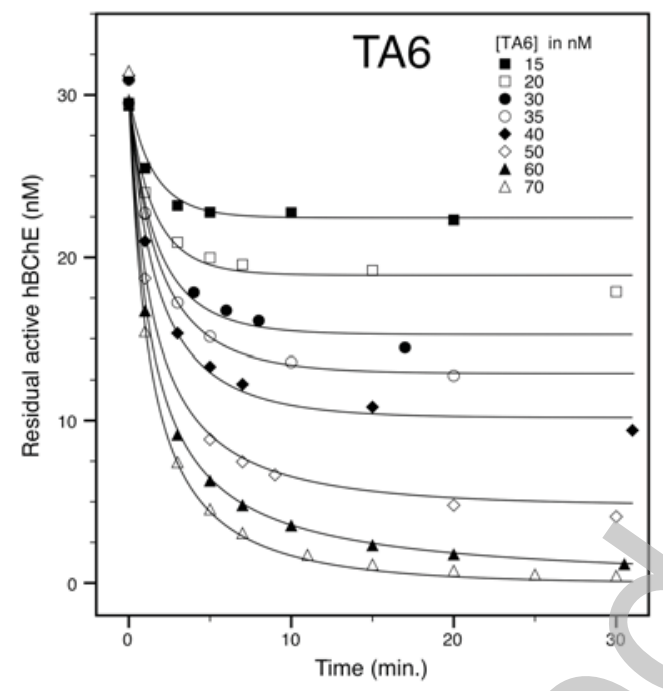

Licenced copy. Copying is not permitted, except with prior permission and as allowed by law. (C) 2009 The Authors Journal compilation (c) 2009 Portland Press Limited 
Fig. 4

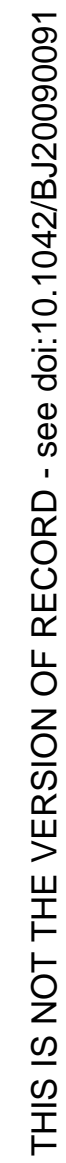
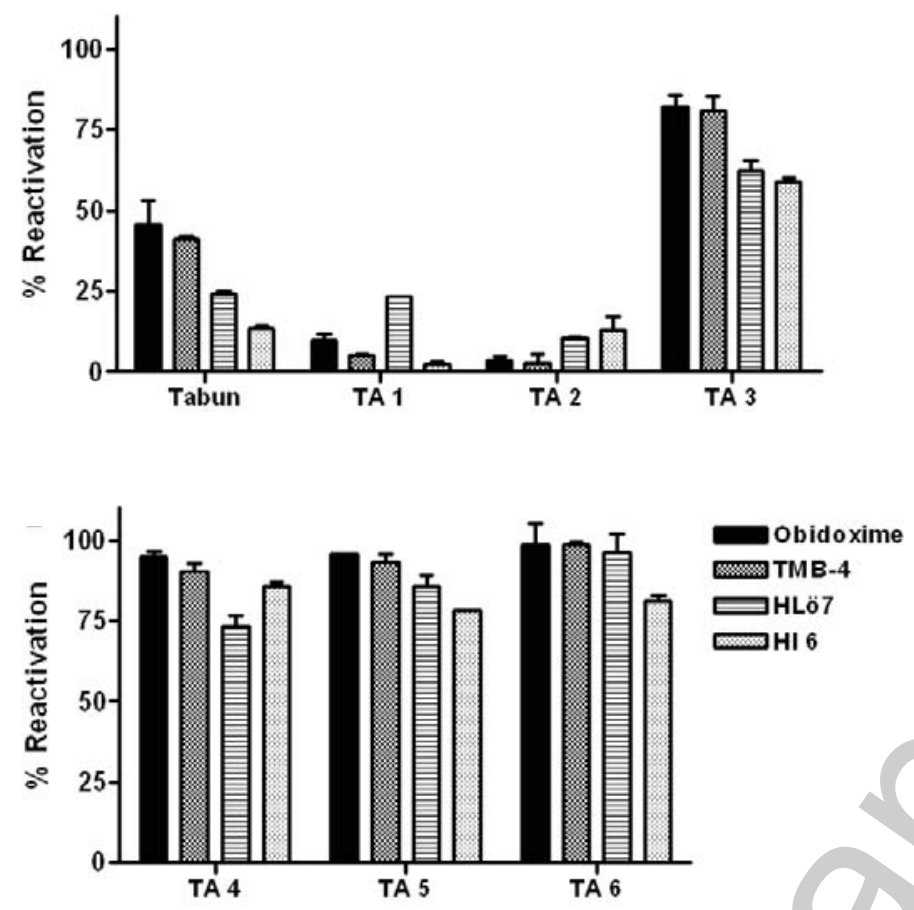
Fig. 5

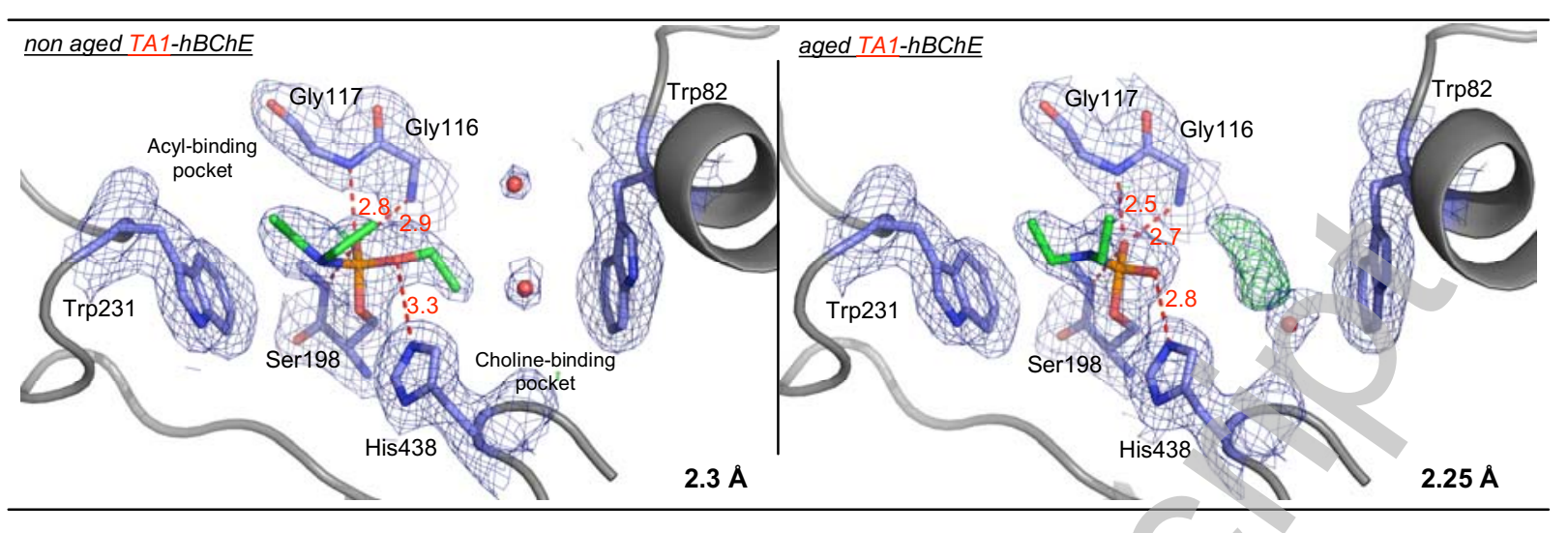

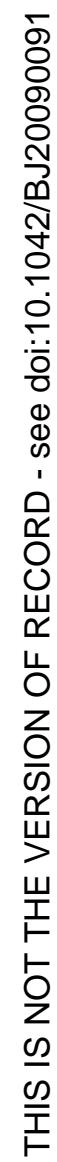
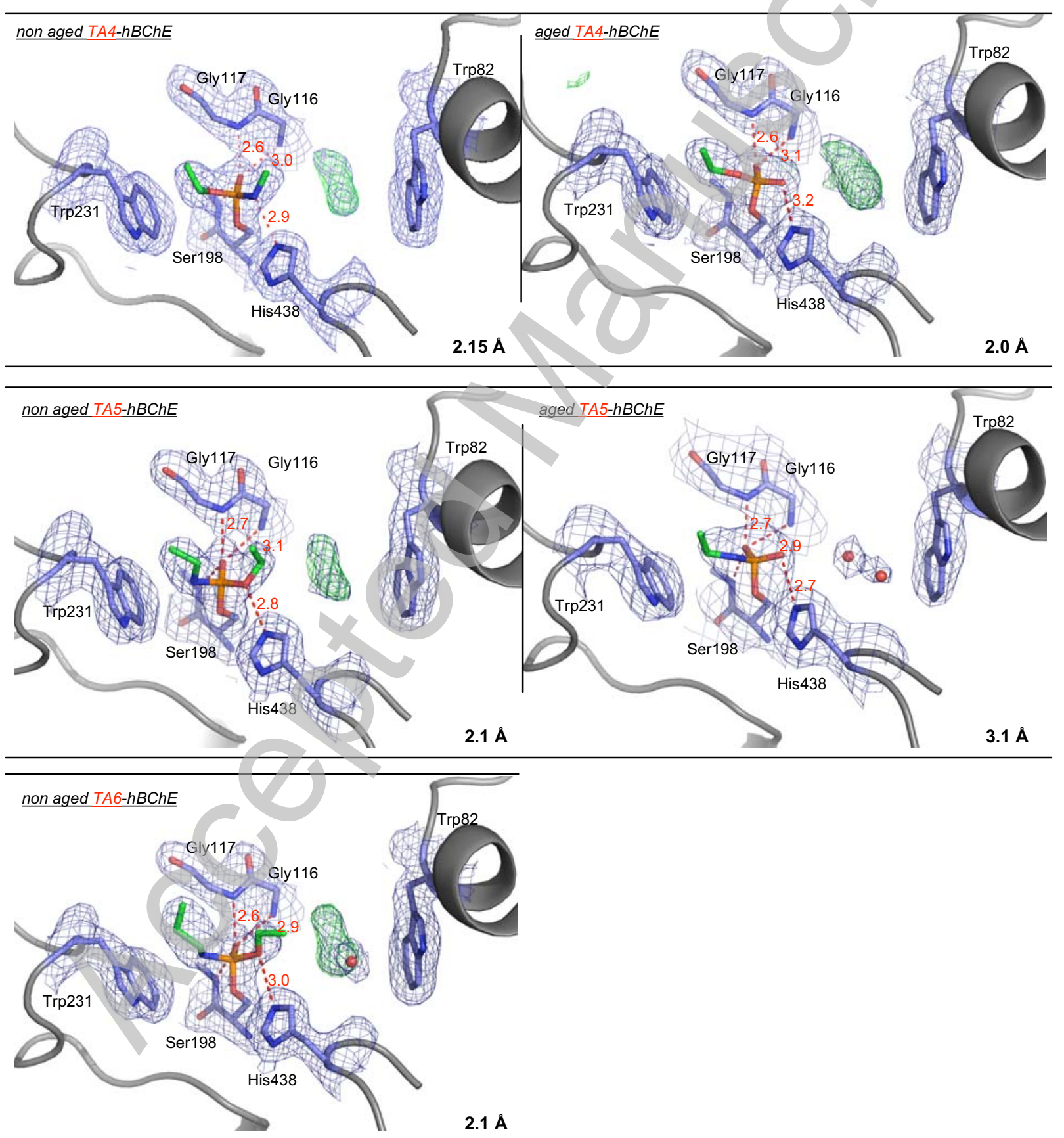

Licenced copy. Copying is not permitted, except with prior permission and as allowed by law. (C) 2009 The Authors Journal compilation (c) 2009 Portland Press Limited 
B) Biochemical Journal Immediate Publication. Published on 15 Apr 2009 as manuscript BJ20090091

Fig. 6

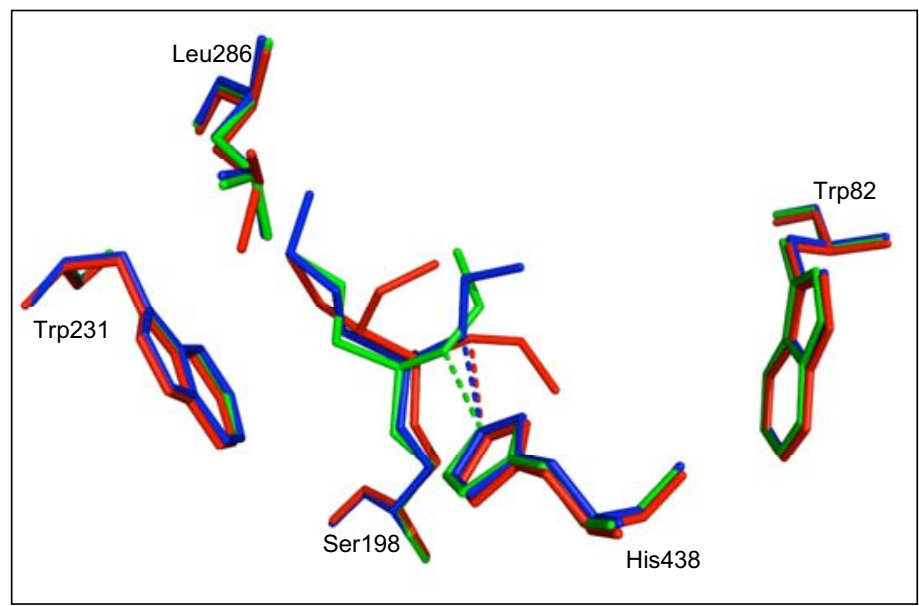

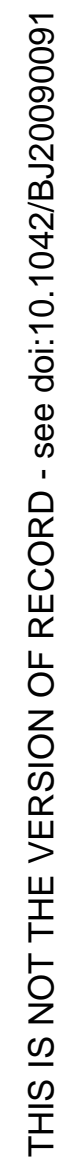


Fig. 7

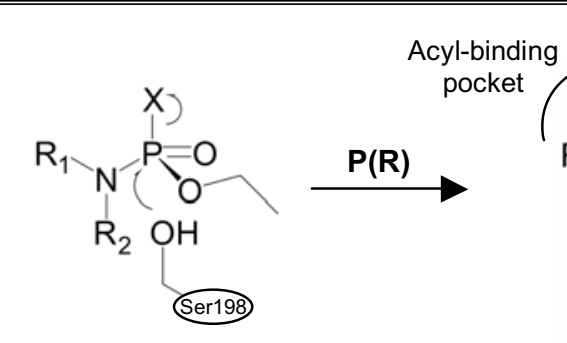

N,N-dialkyl (tabun, TA1)

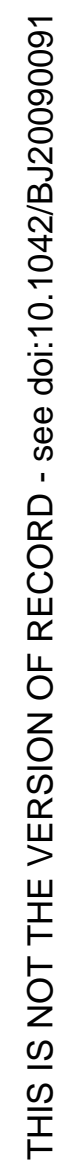

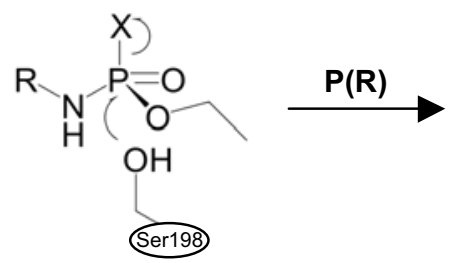

N-monoalkyl (TA5, TA6)

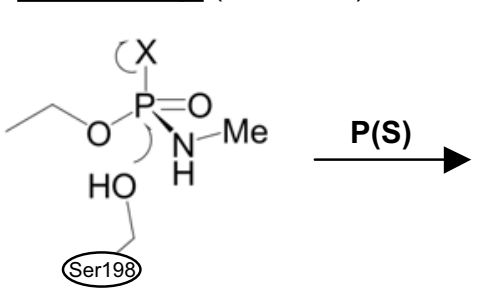

N-monomethyl (TA4)
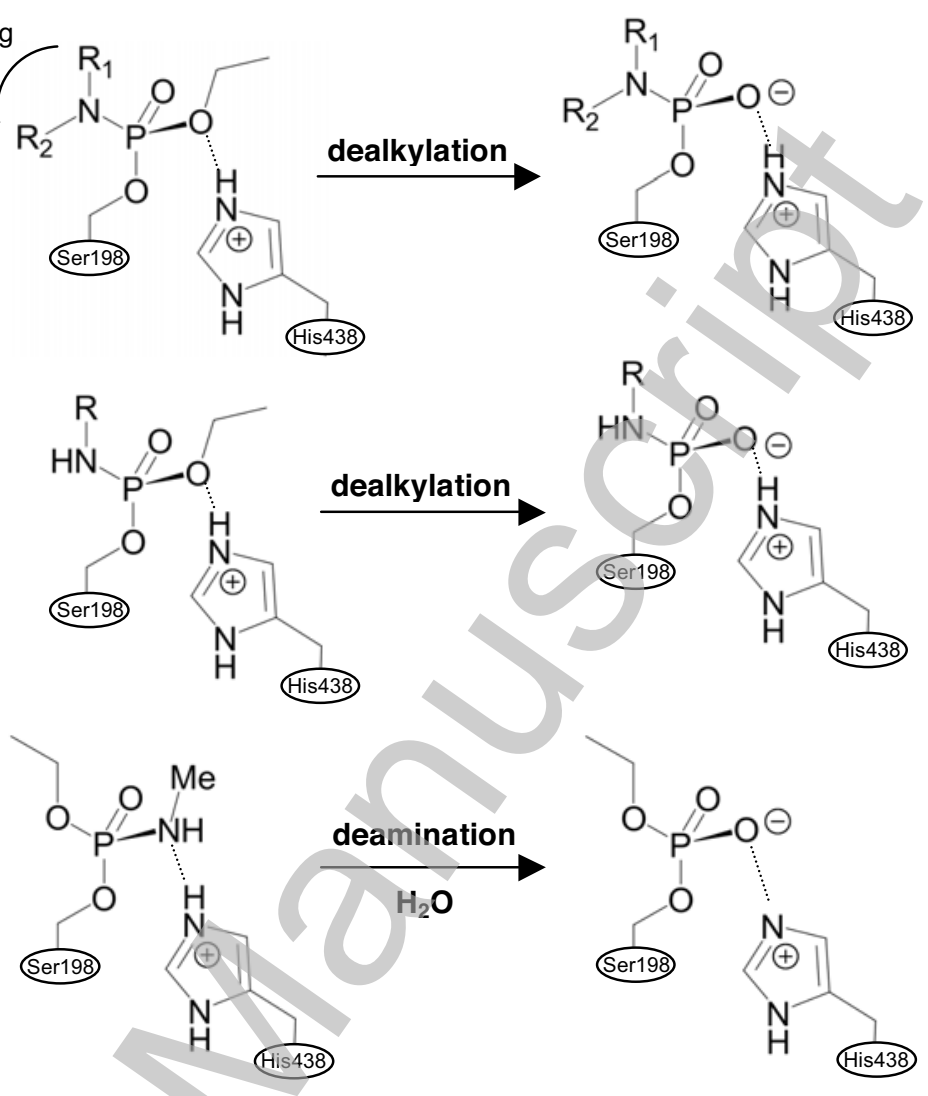